\title{
L'ANALYSE CONCORG SIMULTANÉE : LA MÉTHODE CONCORGS.
}

\author{
${ }^{1}$ GABRIEL KISSITA, ${ }^{2}$ ROGER ARMAND MAKANY, AND ${ }^{3}$ DOMINIQUE MIZÈRE
}

\begin{abstract}
The analysis CONCORG is a method that investigates the link between two multi-tables (partitioned sets of variables measured on the same individuals). It allows to detail the contributions of partial sub-tables forming the two tables. CONCORG is an extension of Concor, which itself is an extension of the inter-battery analysis. This new method performs successive determinations of the solution. In this article, we propose three criteria that enables to simultaneously identify solutions. It happens that the three criteria are equivalent to the solution. In this sense, our method, denoted as CONCORGS, is a generalization of CONCORG. Finally, we establish a fundamental property checked at the optimum these three criteria.
\end{abstract}

Résumé :

L'analyse CONCORG est une méthode recherchant le lien entre deux multitableaux (ensembles des variables partitionnées mesurées sur les mêmes individus). Elle permet de détailler les contributions partielles des sous-tableaux formant les deux tableaux. CONCORG est une extension de CONCOR qui elle-même est un prolongement de l'analyse inter-batterie. C'est une méthode successive dans la détermination de la solution. Dans cet article, nous proposons trois critères généralisant CONCORG. Ces crit'eres permettent de déterminer également des solutions simultanées. Cett méthode est dénommé CONCORGS puisque ces trois critères utilisés sont équivalents à la solution. Enfin, nous établissons une propriété fondamentale vérifiée à l'optimum par ces trois critères.

\section{INTRODUCTION}

L'analyse CONCOR de Lafosse et Hanafi ([5]) est une généralisation de l'analyse Inter-batterie de Tucker ([6]), elle détermine le lien entre un tableau de référence non partitionné $Y$ et un tableau partitionné

$$
X=\left[X_{1}\left|X_{2}\right| \ldots \mid X_{M}\right]
$$

en faisant jouer un rôle privilégié au tableau de référence, les autres $M$ tableaux $X_{i}$ jouant le même rôle entre eux. Elle est fondée sur la

2000 Mathematics Subject Classification. 62H25.

Key words and phrases. Français : multi-tableaux, analyse concor, solution successive, solution simultanée. English : multi-tables, concor analysis, successive solution, simultaneous solution.

Received the 24 November 2009. Revised version received the 27 January 2010. 
${ }^{1}$ GABRIEL KISSITA, ${ }^{2}$ ROGER ARMAND MAKANY, AND ${ }^{3}$ DOMINIQUE MIZÈRE

maximisation de la fonction

$$
f\left(u_{1}, \ldots, u_{M}, v\right)=\sum_{i=1}^{M} \operatorname{cov}^{2}\left(X_{i} u_{i}, Y_{v}\right)
$$

sous les contraintes de norme unité sur les vecteurs $u_{i}(i=1, \ldots, M)$ et $v$. Une solution de ce problème est déterminée de façon successive : c'est-à-dire, après avoir déterminé la solution d'ordre un, la solution d'ordre deux est déterminée en réitérant le procédé donnant la première solution après avoir remplacé les tableaux d'origine par leurs résidus de régression sur la première solution. On réitère cette procédure pour déterminer les solutions d'ordre strictement supérieur à deux. A l'optimum, on montre que la fonction (1) est équivalente à la maximisation de la fonction

$$
g(u, v)=\operatorname{cov}^{2}(X u, Y v)
$$

sous les contraintes de norme unité sur les vecteurs $u$ et $v$. Une généralisation de (1) et (2) est obtenue en partitionnant aussi le tableau de référence $Y$ en sous-tableaux $Y_{j}(j=1, \ldots, N)$. L'analyse ainsi trouvée est nommée analyse CONCORG. Cette méthode est basée sur la maximisation des fonctions (1), (2) et de la fonction

$$
h\left(u, v_{1}, \ldots, v_{N}\right)=\sum_{j=1}^{N} \operatorname{cov}^{2}\left(X u, Y_{j} v_{j}\right)
$$

sous les contraintes de norme unité sur les vecteurs $u$ et $v_{j}(j=$ $1, \ldots, N)$. Une solution successive de CONCORG est donnée dans Kissita et al. ([3]). A l'optimum, on peut écrire la relation fondamentale

$$
\sum_{j=1}^{M} \operatorname{cov}^{2}\left(X_{i} u_{i}, Y v\right)=\operatorname{cov}^{2}(X u, Y v)=\sum_{j=1}^{N} \operatorname{cov}^{2}\left(X u, Y_{j} v_{j}\right) .
$$

Ainsi, dans cet article pour étudier le lien entre deux multi-tableaux, on propose de maximiser trois critères dont on montre (cf. § 4) qu'à l'optimum, ils sont égaux. L'analyse ainsi définie est appelée analyse CONCORGS.

Les données analysées se présentent sous la forme de deux multitableaux : le premier multi-tableaux est une matrice $X=\left[X_{1}\left|X_{2}\right| \ldots \mid X_{M}\right]$ avec $n$ lignes et $m=\sum_{i=1}^{M} m_{i}$ colonnes centrées, partitionnées $M$ en tableaux $X_{i}$ de dimension $n \times m_{i}$ pour $i=1, \ldots, M$; le second est une matrice $Y=\left[Y_{1}\left|Y_{2}\right| \ldots \mid Y_{N}\right]$ avec $n$ lignes et $q=\sum_{j=1}^{N} q_{j}$ colonnes 
centrées, partitionnées en $N$ tableaux $Y_{j}$ de dimension $\mathrm{n} \times q_{j}$. Soit $U_{i}$ (resp. $V_{j}$ ) une matrice de dimension $m_{i} \times r\left(\right.$ resp. $\left.q_{i} \times r\right)$, et $U=\left[U_{1}^{\prime}\left|U_{2}^{\prime}\right| \ldots \mid U_{M}^{\prime}\right]$ (resp. $V=\left[V_{1}^{\prime}\left|V_{2}^{\prime}\right| \ldots \mid V_{N}^{\prime}\right]$ ) la matrice contenant les poids de $X$ (resp. $\left.Y), r \leq \min \left(m_{i}, q_{j}\right), i=1, \ldots, M, j=1, \ldots, N\right)$.

On note par $K_{X Y}=X^{\prime} Y, K_{X_{i} Y}=X_{i}^{\prime} Y$ et $K_{X Y_{j}}=X^{\prime} Y_{j}$ les matrices des inter-covariances entre les tableaux $X$ et $Y, X_{i}$ et $Y, X$ et $Y_{j}$.

On définit par

$$
\operatorname{tr}(\operatorname{Adiag}(A))=\sum_{k=1}^{r} a_{k k}^{2}=\|\operatorname{diag}(A)\|^{2}
$$

où $A=\left[a_{k h}\right], 1 \leq k \leq r$ et $1 \leq h \leq r$ est une matrice carrée, $A^{\prime}$ désigne la transposée de la matrice, $\operatorname{diag}(A)$ la matrice diagonale de $A$ et $I_{r}$ la matrice identité d'ordre $r$.

Les données qui font que la méthode présente un intérêt ont été traitées en grande partie par Vivien (2002) [7]. Mais dans cet article, nous allons reprendre les données entre vins jeunes et vins vieux de Cahors (Laffargue et al.([4]) traitées par Kissita([2]), et Kissita et al ([3]).

Ainsi à la section 2, nous présentons les trois critères de CONCORGS. Enfin à la section 3, nous donnons des algorithmes itératifs permettant de résoudre ces problèmes et donnons une propriété fondamentale vérifiée à la solution par ces critères.

\section{Critères de CONCORGS.}

Pour étudier le lien entre deux multi-tableaux $X$ et $Y$, on propose de maximiser trois critères.

\section{Critère 1 : Maximiser}

$$
f_{1}(U, V)=\operatorname{tr}\left(U^{\prime} X^{\prime} Y V \operatorname{diag}\left(U^{\prime} X^{\prime} Y V\right)\right)=\left\|\operatorname{diag}\left(U^{\prime} K_{X Y} V\right)\right\|^{2}
$$

sous les contraintes $U^{\prime} U=V^{\prime} V=I_{r}$, où $U=\left[U_{1}^{\prime}\left|U_{2}^{\prime}\right| \ldots \mid U_{M}^{\prime}\right]^{\prime}, V=$ $\left[V_{1}^{\prime}\left|V_{2}^{\prime}\right| \ldots \mid V_{N}^{\prime}\right]^{\prime}, X=\left[X_{1}\left|X_{2}\right| \ldots \mid X_{M}\right]$ et $Y=\left[Y_{1}\left|Y_{2}\right| \ldots \mid Y_{N}\right]$ 
${ }^{1}$ GABRIEL KISSITA, ${ }^{2}$ ROGER ARMAND MAKANY, AND ${ }^{3}$ DOMINIQUE MIZÈRE

\section{Critère 2 : Maximiser}

$$
\begin{gathered}
f_{2}\left(U_{1}, \ldots, U_{M}, V_{1}, \ldots, V_{N}\right)=\sum_{i=1}^{M} \operatorname{tr}\left(U_{i}^{\prime} X_{i}^{\prime} Y V \operatorname{diag}\left(U_{i}^{\prime} X_{i}^{\prime} Y V\right)\right) \\
=\sum_{i=1}^{M}\left\|\operatorname{diag}\left(U_{i}^{\prime} K_{X_{i} Y} V\right)\right\|^{2}
\end{gathered}
$$

sous les contraintes $U_{i}^{\prime} U_{i}=V^{\prime} V=I_{r}$, pour $i=1, \ldots, M$.

\section{Critère 3 : Maximiser}

$$
\begin{gathered}
f_{3}\left(U_{1}, \ldots, U_{M}, V_{1}, \ldots, V_{N}\right)=\sum_{j=1}^{N} \operatorname{tr}\left(U^{\prime} X_{i}^{\prime} Y_{j} V_{j} \operatorname{diag}\left(U^{\prime} X^{\prime} Y_{j} V_{j}\right)\right) \\
=\sum_{j=1}^{N}\left\|\operatorname{diag}\left(U^{\prime} K_{X Y_{j}} V_{j}\right)\right\|^{2}
\end{gathered}
$$

sous les contraintes $U^{\prime} U=V_{j}^{\prime} V_{j}=I_{r}$, pour $j=1, \ldots, N$.

Ces trois critères sont égaux à l'optimum (et égaux au critère optimisé dans CONCORG quand $r=1$ ) rappelons-le, voir le paragraphe 4 .

2. Maximisation Des FOnCtions $f_{\alpha}(\alpha=1,2,3)$

Pour maximiser $f_{\alpha}(\alpha=1,2,3)$ sous les contraintes $U^{\prime} U=V^{\prime} V=I_{r}$, $U_{i}^{\prime} U_{i}=V^{\prime} V=I_{r}$ et $U^{\prime} U=V_{j}^{\prime} V_{j}=I_{r}$ respectivement pour $i=1, \ldots, M$ et $j=1, \ldots, N$ deux algorithmes itératifs associés à 1 et 2 sont donnés, celui de 3 est similaire à 2 en intervertissant les rôles de $i$ et $j$. Ces algorithmes procèdent par la mise à jour itérative de $U$ et $V$. La monotonie revient à montrer la croissance des fonctions $f_{\alpha}$ d'abord par rapport à $U$ et après par rapport à $V$.

2.1. Maximisation de $f_{1}$. Pour construire les mises à jour $U^{*}$ et $V^{*}$ des matrices $U$ et $V$, on choisit aléatoirement des matrices vérifiant les contraintes $U^{\prime} U=V^{\prime} V=I_{r}$. Cette construction va se faire alternativement et en trois étapes. La première étape est consacrée à la maximisation de $f_{1}$ par rapport à $U$, la seconde étape consiste à maximiser $f_{1}$ par rapport à $V$ et la troisième étape est réservée à la monotonie de l'algorithme. 


\section{Première étape : détermination de la mise à jour $U^{*}$.}

Pour déterminer la mise à jour $U^{*}$ dans le problème : maximiser $f_{1}(U, V)=\operatorname{tr}\left(U^{\prime} X^{\prime} Y V \operatorname{diag}\left(U^{\prime} X^{\prime} Y V\right)\right)$, sous les contraintes $U^{\prime} U=$ $V^{\prime} V=I_{r}$. On procède ainsi : en notant $K_{X Y}=X^{\prime} Y$ rappelons le, on écrit la fonction $f_{1}(U, V)=\operatorname{tr} U^{\prime} T_{U V}$ avec $T_{U V}=K_{X Y} V \operatorname{diag}\left(U^{\prime} K_{X Y} V\right)$. On prend la décomposition en valeurs singulières de $T_{U V}=P D Q^{\prime}$ où $P^{\prime} P=Q^{\prime} Q=Q Q^{\prime}=I_{r}$. La mise à jour de $U$ est donnée par la matrice $U^{*}=P Q^{\prime}$. Pour la preuve de ce résultat voir Cliff ([1]).

\section{Deuxième étape : détermination de la mise à jour $V^{*}$.}

Pour trouver la mise à jour de $V$ notée $V^{*}$, on écrit $f_{1}$ sous la forme

$$
f_{1}(U, V)=\operatorname{tr}\left(V^{\prime} K_{Y X} U \operatorname{diag}\left(U^{\prime} K_{X Y} V\right)\right)=\operatorname{tr} V^{\prime} H_{U V}
$$

où $\left.H_{U V}=K_{X Y} U \operatorname{diag} U^{\prime} K_{X Y} V\right)$ et $U=U^{*}$ déterminée à la première étape. En procédant comme à l'étape 1 , on trouve $V^{*}=L G^{\prime}$ la mise à jour de $V$ avec $H_{U V}=L \Delta G^{\prime}$ la décomposition en valeurs singulières de $H_{U V}$ où $L^{\prime} L=G^{\prime} G=G G^{\prime}=I_{r}$.

\section{Troisième étape : monotonie de l'algorithme.}

Nous ne montrons la monotonie de l'algorithme que par rapport à $U$, sachant que celle de $V$ se démontre de la même manière en échangeant seulement les rôles de $U$ et $V$. Il est donc question de montrer que

$$
f_{1}(U, V) \leq f_{1}\left(U^{*}, V\right) \leq f_{1}\left(U^{*}, V^{*}\right) .
$$

Pour établir cette monotonie par rapport à $U$, il suffit de montrer que $f_{1}(U, V) \leq f_{1}\left(U^{*}, V\right)$. En effet, à l'aide de la matrice $U^{* \prime} T_{U V}=$ $U^{* \prime} K_{X Y} V \operatorname{diag}\left(U^{\prime} K_{X Y} V\right)$, on peut en tirer le $k^{i e ̀ m e}$ élément diagonal

$$
u_{k}^{* \prime} K_{X Y} v_{k} u_{k}^{\prime} K_{X Y} v_{k}=u_{k}^{* \prime} G_{X Y k} u_{k}
$$

où $u_{k}=\left[u_{1 k}^{\prime}|\ldots| u_{i k}^{\prime}|\ldots| u_{M k}^{\prime}\right]^{\prime}$ et $u_{k}=\left[v_{1 k}^{\prime}|\ldots| v_{i k}^{\prime}|\ldots| v_{N k}^{\prime}\right]^{\prime}$ sont respectivement les $k^{i e ̀ m e s}$ vecteurs blocs colonnes des matrices $U$ et $V$, et $G_{X Y k}=K_{X Y} v_{k} v_{k}^{\prime} K_{Y X}$ une matrice symétrique semi-définie positive. Par conséquent :

$$
\left\|G_{X Y k}^{1 / 2} u_{k}-G_{X Y k}^{1 / 2} u_{k}^{*}\right\|^{2} \geq 0 .
$$

La relation (8) est équivalente à la relation ci-dessous

$$
u_{k}^{\prime} G_{X Y k} u_{k}+u_{k}^{* \prime} G_{X Y} u_{k}^{*} \geq 2 u_{k}^{* \prime} K_{X Y} v_{k} u_{k}^{\prime} K_{X Y} v_{k}
$$


ou encore

$$
u_{k}^{\prime} K_{X Y} v_{k} u_{k}^{\prime} K_{X Y} v_{k}+u_{k}^{* \prime} K_{X Y} v_{k} u_{k}^{* \prime} K_{X Y} v_{k} \geq 2 u_{k}^{* \prime} K_{X Y} v_{k} u_{k}^{\prime} K_{X Y} v_{k}
$$

En sommant (10) par rapport à $k$, on trouve la relation équivalente suivante

$\operatorname{tr}\left(U^{\prime} K_{X Y} V \operatorname{diag}\left(U^{\prime} K_{X Y} V\right)+\operatorname{tr}\left(U^{* \prime} K_{X Y} V \operatorname{diag}\left(U^{* \prime} K_{X Y} V\right)\right) \geq 2 \operatorname{tr}\left(U^{*} K_{X Y} V \operatorname{diag}\left(U^{\prime} K_{X Y} V\right)\right.\right.$.

En se référant à Cliff ([1]), on a, à partir de la mise à jour $U^{*}$ la relation

$$
\operatorname{tr}\left(U^{* \prime} K_{X Y} V \operatorname{diag}\left(U^{\prime} K_{X Y} V\right)\right) \geq \operatorname{tr}\left(U^{\prime} K_{X Y} V \operatorname{diag}\left(U^{\prime} K_{X Y} V\right)\right) .
$$

En combinant (11) et (12), on trouve la relation recherchée ci-dessous

$$
\operatorname{tr}\left(U^{* \prime} K_{X Y} V \operatorname{diag}\left(U^{* \prime} K_{X Y} V\right)\right) \geq \operatorname{tr}\left(U^{\prime} K_{X Y} V \operatorname{diag}\left(U^{\prime} K_{X Y} V\right)\right) .
$$

Ce qui permet d'écrire $f_{1}\left(U^{*}, V\right) \geq f_{1}(U, V)$. D'où la monotonie de l'algorithme par rapport à $U$. Le résumé de l'algorithme itératif de détermination de la solution concernant la maximisation de $f_{1}$ sous les contraintes $U^{\prime} U=V^{\prime} V=I_{r}$ est placé en annexe 1 .

On peut donc dire que les mises à jour de $U$ et de $V$ font croître monotonement la fonction $f_{1}$. La fonction étant bornée, continue et croissante monotone en particulier sur l'ensemble formé par les vecteurs normés constitués par les colonnes des matrices $U$ et $V$, l'algorithme converge. Le choix particulier de $U=U^{*}$ implique que $U^{* \prime} T_{U V}=$ $Q P^{\prime} P D Q^{\prime}=Q D Q^{\prime}$ est une matrice symétrique semi-définie positive, et donc $D$ contient des valeurs singulières positives ou nulles.

2.2. Maximisation de $f_{2}$. Pour construire les mises à jour $U_{i}^{*}(i=$ $1, \ldots, M)$ et $V^{*}$ trois étapes sont aussi considérées. La première est donc relative à la maximisation de $f_{2}$ par rapport à $U_{i}(i=1, \ldots, M)$, la seconde étape est réservée à $V$ et la troisième concerne la monotonie de $f_{2}$.

\section{Première étape : détermination de la mise à jour $U_{i}^{*}$.}

Le problème à résoudre est la maximisation de $f_{2}$ sous les contraintes $U_{i}^{\prime} U_{i}=V^{\prime} V=I_{r}(i=1, \ldots, M)$, ce qui est équivalent à la maximisation de

$$
\operatorname{tr}\left(U_{i}^{\prime} K_{X Y} V \operatorname{diag}\left(U_{i}^{\prime} K_{X Y} V\right)\right)
$$


sous les mêmes contraintes et où $K_{X_{i} Y}=X_{i}^{\prime} Y$. Ainsi à la première étape, on détermine la mise à jour $U_{i}^{*}(i=1, \ldots, M)$. Pour cela, on écrit

$$
\operatorname{tr}\left(U_{i}^{\prime} K_{X Y} V \operatorname{diag}\left(U_{i}^{\prime} K_{X Y} V\right)\right)=\operatorname{tr} U_{i}^{\prime} T_{U_{i} V}
$$

où

$$
T_{U_{i} V}=K_{X_{i} Y} V \operatorname{diag}\left(U_{i}^{\prime} K_{X_{i} Y} V\right)
$$

est une matrice de dimension $m_{i} \times r(i=1, \ldots, M)$. En faisant la décomposition en valeurs singulières de

$$
T_{U_{i} V}=P_{i} D_{i} Q_{i}^{\prime}
$$

avec $P_{i}^{\prime} P_{i}=Q_{i}^{\prime} Q_{i}=I_{r}$. La mise à jour de $U_{i}$ est $U_{i}^{*}=P_{i} Q_{i}^{\prime}$ $(i=1, \ldots, M)$ (voir Cliff [1]).

\section{Deuxième étape : détermination de la mise à jour $V^{*}$.}

A l'étape 2, on détermine la mise à jour de $V$. Pour construire cette mise à jour de $V$, on remarque que

$$
f_{2}=\operatorname{tr} V^{\prime} \sum_{i=1}^{M} K_{Y X_{i}} U_{i} \operatorname{diag}\left(U_{i}^{\prime} K_{X_{i} Y} V\right)=\operatorname{tr} V^{\prime} H_{U V}
$$

où

$$
H_{U V}=\sum_{i=1}^{M} K_{Y X_{i}} U_{i} \operatorname{diag}\left(U_{i}^{\prime} K_{X_{i} Y} V\right)
$$

est une matrice de dimension $q \times r$ et où $U_{i}=U_{i}^{*}$ trouvée à l'étape 1. La décomposition en valeurs singulières de $H_{U V}=L \Delta G^{\prime}$ avec $L^{\prime} L=G^{\prime} G=G^{\prime} G=I_{r}$ conduit à la mise à jour $V^{*}=L G^{\prime}$.

\section{Troisième étape : monotonie de l'algorithme.}

Après avoir trouvé les mises à jour par rapport à $U_{i}(i=1, \ldots, M)$ et à $V$, on peut maintenant établir de la même manière que précédemment la monotonie de l'algorithme relativement à $U_{i}(i=1, \ldots, M)$. En effet, considérons le $k^{i e ̀ m e}$ élément diagonal de la matrice

$$
U_{i}^{* \prime} T_{U_{i} V}=U_{i}^{* \prime} K_{X_{i} Y} V \operatorname{diag}\left(U_{i}^{\prime} K_{X_{i} Y} V\right)
$$

défini par le scalaire

$$
u_{i k}^{* \prime} K_{X_{i} Y} v_{k} u_{i k}^{\prime} K_{X_{i} Y} v_{k}=u_{i k}^{* \prime} G_{X_{i} Y k} u_{i k}
$$

où $u_{i k}$ et $v_{k}$ sont respectivement les $k^{i e ̀ m e s}$ colonnes des matrices $U_{i}$ et $V$, avec

$$
G_{X_{i} Y k}=K_{X_{i} Y} v_{v} v_{k}^{\prime} K_{X_{i} Y}
$$


${ }^{1}$ GABRIEL KISSITA, ${ }^{2}$ ROGER ARMAND MAKANY, AND ${ }^{3}$ DOMINIQUE MIZÈRE

une matrice symétrique semi-définie positive. Il en résulte pour $i=$ $1, \ldots, M, l$ l'inégalité ci-dessous

$$
\left\|G_{X_{i} Y k}^{1 / 2} u_{i k}-G_{X_{i} Y k}^{1 / 2} u_{i k}^{*}\right\|^{2} \geq 0 .
$$

(14) est équivalente à

$$
u_{i k}^{\prime} G_{X_{i} Y k} u_{i k}+u_{i k}^{* \prime} G_{X_{i} Y k} u_{i k}^{*} \geq 2 u_{i k}^{* \prime} G_{X_{i} Y} u_{i k}
$$

ou encore

$$
u_{i k}^{\prime} K_{X_{i} Y} v_{k} u_{i k}^{\prime} K_{X_{i} Y} v_{k}+u_{i k}^{* \prime} K_{X_{i} Y} v_{k} u_{i k}^{* \prime} K_{X_{i} Y} v_{k} \geq 2 u_{i k}^{*^{\prime}} K_{X_{i} Y} v_{k} u_{i k}^{\prime} K_{X_{i} Y} v_{k}
$$

En sommant (16) sur $k$, il en découle pour $i=1, \ldots, M$, la relation suivante :

$$
\begin{gathered}
\operatorname{tr}\left(U_{i}^{\prime} K_{X_{i} Y} V \operatorname{diag}\left(U_{i}^{\prime} K_{X_{i} Y} V\right)\right)+\operatorname{tr}\left(U_{i}^{* \prime} K_{X_{i} Y} \operatorname{diag}\left(U_{i}^{* \prime} K_{X_{i} Y} V\right)\right) \\
\geq 2 \operatorname{tr}\left(U_{i}^{* \prime} K_{X_{i} Y} V \operatorname{diag}\left(U_{i}^{\prime} K_{X_{i} Y} V\right)\right)
\end{gathered}
$$

$U_{i}^{*}$ étant la mise à jour de $U_{i}$, selon Cliff ([1]), on a la relation suivante

$$
\operatorname{tr}\left(U_{i}^{* \prime} K_{X_{i} Y} V \operatorname{diag}\left(U_{i} K_{X_{i} Y} V\right)\right) \geq \operatorname{tr}\left(U_{i}^{* \prime} K_{X_{i} Y} V \operatorname{diag}\left(U_{i} K_{X_{i} Y} V\right)\right)
$$

En mettant (17) dans (18), on trouve la relation recherchée

$$
\operatorname{tr}\left(U_{i}^{* \prime} K_{X_{i} Y} V \operatorname{diag}\left(U_{i}^{*} K_{X_{i} Y} V\right)\right) \geq \operatorname{tr}\left(U_{i}^{* \prime} K_{X_{i} Y} V \operatorname{diag}\left(U_{i} K_{X_{i} Y} V\right)\right)
$$

Enfin en sommant (19) sur $i$, on trouve $f_{2}\left(U^{*}, V\right) \geq f_{2}(U, V)$ où $U=\left[U_{1}^{\prime}\left|U_{2}^{\prime}\right| \ldots \mid U_{M}^{\prime}\right]^{\prime}$. On peut donner un résumé de l'algorithme itératif permettant de déterminer la solution au problème de maximisation de $f_{2}$ sous les contraintes $U_{i}^{\prime} U_{i}=V^{\prime} V=I_{r}(i=1, \ldots, M)$ en annexe 2 .

2.3. Algorithme pour la maximisation de $f_{3}$. Pour maximiser

$$
f_{3}=\sum_{j=1}^{N} \operatorname{tr}\left(U^{\prime} K_{X Y_{j}} V_{j} \operatorname{diag}\left(U^{\prime} K_{X Y_{j}} V_{j}\right)\right.
$$

avec les contraintes $U^{\prime} U=V_{j}^{\prime} V_{j}=I_{r}(j=1, \ldots, N)$, nous procédons comme pour la maximisation de $f_{2}$ sous les contraintes $U_{i}^{\prime} U_{i}=V^{\prime} V=$ $\left.I_{r} i=1, \ldots, M\right)$ en intervertissant les rôles de $U_{i}$ et $V_{i}$, et ensuite de $U$ et $V$. Par conséquent, on trouve le résumé de l'algorithme itératif permettant de maximiser $f_{3}$ sous les contraintes ci-dessus en annexe 3 . 


\section{RElation FONDAMENTAle vÉRIFiÉE PAR CONCORGS}

La relation que nous allons donner dans la propriété suivante est une des caractéristique des méthodes CONCORG dans leur ensemble. Ici, cette relation est une extension de (4) dans CONCORG $(r=1)$. A l'optimum, on a la relation

$$
\begin{gathered}
\sum_{i=1}^{M} \operatorname{tr}\left(U_{i}^{\prime} K_{X_{i}} V \operatorname{diag}\left(U_{i}^{\prime} K_{X_{i}} V\right)\right)= \\
\operatorname{tr}\left(U^{\prime} K_{X Y} V \operatorname{diag}\left(U^{\prime} K_{X Y} V\right)\right)=\sum_{j}^{N} \operatorname{tr}\left(U^{\prime} K_{X Y_{j}} V_{j} \operatorname{diag}\left(U^{\prime} K_{X Y_{j}} V_{j}\right)\right)
\end{gathered}
$$

La preuve de cette propriété est immédiate, car elle est basée sur la relation (4) donnée en dimension un. En effet, on sait que :

$$
\begin{gathered}
\sum_{i=1}^{M} u_{i k}^{\prime} K_{X_{i} Y} v_{k} u_{i k}^{\prime} K_{X_{i} Y} v_{k}=u_{k}^{\prime} K_{X Y} v_{k} u_{k}^{\prime} K_{X Y} v_{k} \\
=\sum_{j=1}^{N} u_{k}^{\prime} K_{X Y_{j}} v_{j k} u_{k}^{\prime} K_{X Y_{j}} v_{j k},
\end{gathered}
$$

où $u_{i k}, u_{k}, v_{j k}$ et $v_{k}$ sont respectivement les $k^{i e ̀ m e s}$ colonnes des matrices $U_{i}, U, V_{j}$ et de $V$. En sommant par rapport à $k$, la relation (21), on trouve la relation (20).

\section{Application}

Pour établir la pertinence de la démarche CONCORGS par rapport à l'approche successive, nous reprenons les données entre vins jeunes et vins vieux de Cahors (Laffargue et al. ([4]) rappelons-le qui ont aussi été traitées par Kissita ([2]), Kissita et al. ([3]), Lafosse et Ten Berge ([5]). Ces données sont issues d'une expérimentation conduite par le syndicat de vins de Cahors. Différents tableaux sont constitués, chacun d'eux étant relatif à une année donnée (1983, 1985, 1988, 1989, 1993). Les 30 lignes de chaque tableau désignent $5 \times 6$ vins différents, un vin étant défini par un greffon donné associé à un porte-greffe donné. Les 5 porte-greffes différents et les 6 greffons possibles définissent alors 30 vins, rangés dans un même ordre quelque soit le tableau. Chaque année, 8 variables sont mesurées au moment de la récolte, puis 5 ans après quand le vin a vieilli. Elles définissent les 8 colonnes de chacun des 10 tableaux considérés ( 5 tableaux vins jeunes et 5 tableaux vins 
${ }^{1}$ GABRIEL KISSITA, ${ }^{2}$ ROGER ARMAND MAKANY, AND ${ }^{3}$ DOMINIQUE MIZÈRE

vieux). Il s'agit des mesures physico-chimiques suivantes : anthocyane (an), indice de réfraction du rouge (ro), indice de réfraction du jaune (ja), indice de folin (fo), degré en alcool (da), extrait sec (es), acidité totale (at), acidité volatile (av).

Le tableau ci-dessous donne les valeurs des critères CONCORG (méthode successive), CONCORGS (méthode simultanée) pour les 8 solutions.

\begin{tabular}{|l|l|l|l|l|l|l|l|l|}
\hline Solution & 1 & 2 & 3 & 4 & 5 & 6 & 7 & 8 \\
\hline CONCORG & 64.45 & 24.07 & 9.16 & 1.55 & 0.67 & 0.21 & 0.00 & 0.00 \\
\hline CONCORGS & 69.7 & 25.5 & 2.29 & $1 ? 17$ & 1.12 & 0.89 & 0.2 & 0.00 \\
\hline
\end{tabular}

Les valeurs des critères pour les méthodes successive (CONCORG) et simultanée (CONCORGS) sont respectivement 100.11 et 100.87.

En plus les deux premières solutions sont respectivement pour la méthode successive et simultanée $88.52 \%$ et $95.2 \%$. On constate que les valeurs de la méthode simultanée sont nettement supérieures par rapport aux valeurs de la méthode successive. C'est le premier algorithme qui a conduit au meilleur maximum.

Toutes ces méthodes ont un même objectif, on peut donc affirmer que pour ce genre des données, l'utilisateur peut choisir n'importe quelle méthode.

\section{CONCLUSION}

Nous venons là de définir une méthode simultanée de détermination de la solution de CONCORG. Ensuite, nous avons proposé des algorithmes convergents vers des solutions locales. Mais, pour obtenir des solutions globales, on tourne les algorithmes en adoptant plusieurs initialisations, et on choisit les plus grandes valeurs des fonctions, ce sont ces valeurs qui correspondent à la solution. Enfin, nous avons établi la relation fondamentale vérifiée à l'optimum par la solution. 
L'ANALYSE CONCORG SIMULTANÉE : LA MÉTHODE CONCORGS.

\section{Annexes}

\subsection{Annexe 1 : algorithme maximisant de $f_{1}$.}

1) Choisir $U, V$ (exemple aléatoirement, telles que $U^{\prime} U=V^{\prime} V=$ $I_{r}$ ), et $\varepsilon$ (exemple, 0.00001).

2) Détermination des mises à jour.

A) Mise à jour de $U$.

1) Calculer $T_{U V}=K_{X Y} V \operatorname{diag}\left(U^{\prime} K_{X Y} V\right)$.

2) Faire la décomposition en valeurs singulières de $T_{U V}=$ $P D Q^{\prime}$.

3) Poser pour mise à jour de $U$, la matrice $U^{*}=P Q^{\prime}$.

B) Mise à jour $V$.

1) Calculer $H_{U V}=K_{Y X} U \operatorname{diag}\left(U^{\prime} K_{X Y} V\right)$, où $U=U^{*}$ trouvée précédemment.

2) Effectuer la décomposition en valeurs singulières de $H_{U V}=L \Delta G^{\prime}$.

3) Poser pour mise à jour de $V$, la matrice $V^{*}=L G^{\prime}$.

3) Si $f_{1}\left(U^{*}, V^{*}\right)-f_{1}(U, V) \leq \varepsilon$, alors l'algorithme a convergé, sinon $U^{*}=U$ et $V^{*}=V$ et aller à 2 .

\subsection{Annexe 2 : algorithme maximisant $f_{2}$.}

1) Choisir $U, V$ (exemple aléatoirement, telles que $U_{i}^{\prime} U_{i}=V^{\prime} V=$ $I_{r}$ ), et $\varepsilon$ (exemple, 0.00001).

2) Recherche des mises à jour.

A) Mise à jour de $U_{i}(i=1, \ldots, M)$.

1) Calculer $T_{U_{i} V}=K_{X_{i} Y} V \operatorname{diag}\left(U_{i}^{\prime} K_{X_{i} Y} V\right)$ pour $(i=1, \ldots, M)$.

2) Faire la décomposition en valeurs singulières de $T_{U_{i} V}=$ $P_{i} D_{i} Q_{i}^{\prime}$ pour $(i=1, \ldots, M)$.

3) Poser pour mise à jour de $U_{i}$, la matrice $U_{i}^{*}=P_{i} Q_{i}^{\prime}$ pour $(i=1, \ldots, M)$.

4) Poser $U^{*}=\left[U_{1}^{* \prime}\left|U_{2}^{* \prime}\right| \ldots \mid U_{M}^{* \prime}\right]^{\prime}$.

B) Mise à jour $V$.

1) Calculer $H_{U V}=\sum_{i=1}^{M} K_{Y X_{i}} U_{i} \operatorname{diag}\left(U_{i}^{\prime} K_{X_{i} Y} V\right)$, où $U_{i}=$ $U_{i}^{*}$ déterminée au point $(2)$.

2) Effectuer la décomposition en valeurs singulières de $H_{U V}=L \Delta G^{\prime}$.

3) Poser pour mise à jour de $V$, la matrice $V^{*}=L G^{\prime}$.

3) Si $f_{2}\left(U^{*}, V^{*}\right)-f_{2}(U, V) \leq \varepsilon$, alors l'algorithme a convergé, sinon $U^{*}=U$ et $V^{*}=V$ et aller à 2 . 
${ }^{1}$ GABRIEL KISSITA, ${ }^{2}$ ROGER ARMAND MAKANY, AND ${ }^{3}$ DOMINIQUE MIZÈRE

\subsection{Annexe 3 : algorithme maximisant $f_{3}$.}

1) Choisir $U, V$ (exemple aléatoirement, telles que $U^{\prime} U=V_{j}^{\prime} V_{j}=$ $I_{r}$ ), et $\varepsilon$ (exemple, 0.00001 ).

2) Recherche des mises à jour.

A) Mise à jour de $U$.

1) Calculer $T_{U V}=\sum_{j=1}^{N} K_{X Y_{j}} V_{j} \operatorname{diag}\left(U^{\prime} K_{X Y_{j}} V_{j}\right)$.

2) Faire la décomposition en valeurs singulières de $T_{U}=$ $P D Q$.

3) Poser pour mise à jour de $U_{i}$, la matrice $U_{i}^{*}=P_{i} Q_{i}^{\prime}$ pour $(i=1, \ldots, M)$.

* Poser $U^{*}=P Q^{\prime}$.

B) Mise à jour $V_{j}$ pour $j=1, \ldots, N$.

1) Calculer pour $j=1, \ldots, N, H_{U V_{j}}=K_{Y_{j} X} U \operatorname{diag}\left(U^{\prime} K_{X Y} V_{j}\right)$, où $U=U^{*}$.

2) Effectuer la décomposition en valeurs singulières de $H_{U V_{j}}=L_{j} \Delta_{j} G_{j}^{\prime}$ pour $j=1, \ldots, N$.

3) Poser pour mise à jour de $V_{j}(j=1, \ldots, N)$ la matrice $V_{j}^{*}=L_{j} G_{j}^{\prime}$.

4) Poser $V^{*}=\left[V_{1}^{* \prime}\left|V_{2}^{* \prime}\right| \ldots \mid V_{N}^{* \prime}\right]^{\prime}$.

3) Si $f_{3}\left(U^{*}, V^{*}\right)-f_{3}(U, V) \leq \varepsilon$, alors l'algorithme a convergé, sinon $U^{*}=U$ et $V^{*}=V$ et aller à 2 . 


\section{REFERENCES}

[1] Cliff, N.(1966). Orthogonal rotation to congruence. Psychometrika 31, $33-42$.

[2] Kissita, G., 2003. Les analyses canoniques généralisées avec tableau de référence généralisé : éléments théoriques et appliqués. Thèse, Université Paris- Dauphine.

[3] Kissita, G., Cazes, P., Hanafi, M., Lafosse, R., 2004. Deux méthodes d'analyse factorielle du lien entre deux tableaux de variables partitionnées. Rev, Statist. Appl. 52 (3), 73-92.

[4] Laffargue et coll. (1983-93). Expérimentation porte-greffes, greffons du cépage Cot. Maison des vins de Cahors. Ch. Agr. du Lot. CETA de Luzech. Lafosse, R., Hanafi, M., 1997. Concordance d'un tableau avec K tableaux : définition des $\mathrm{K}+1$ uplés synthétiques. Rev. Statist. Appl. 45 (4), 111-126.

[5] Lafosse, R., Ten Berge, J.M.S., 2006. A simultaneous CONCOR algorithm for the analysis of two partitioned matrices. Computational Statistics and Data Analysis 50, 2529-2535.

[6] Tucker, L.R., 1958. An interbattery method for factor invariance. Psychometrika 23, 111-136.6.

[7] Vivien, M., 2002. Approches PLS linéaires et non linéaires pour la modélisation de multi-tableaux : théorie et applications. Thèse, Université Montpellier I.

${ }^{1}$ ISG, Université Marien Ngouabi Brazzaville-Congo, Gakissita@yahoo.fr

${ }^{2}$ FSE, Université Marien Ngouabi Brazzaville-Congo, Rmakany@yahoo.fr

${ }^{3}$ FS, Université Marien Ngouabi, Brazzaville-Congo, DMizère@yahoo.fr 